\title{
Blood Pressure is Associated with Rapid Kidney Function Decline in a Very Elderly Hypertensive Chinese Population
}

This article was published in the following Dove Press journal:

Clinical Interventions in Aging

\section{Kunhao Bai ${ }^{1,2, *}$ \\ Rui Chen ${ }^{2, *}$ \\ Fanghong $\mathrm{Lu}^{3}$ \\ Yingxin $\mathrm{Zhao}^{3}$ \\ Yujing $\operatorname{Pan}^{4}$ \\ Fang Wang ${ }^{2}$ \\ Luxia Zhang $2,5,6$}

'Department of Endoscopy, Sun Yat-sen University Cancer Center, State Key Laboratory of Oncology in South China, Collaborative Innovation Center for Cancer Medicine, Guangzhou, Guangdong, People's Republic of China; ${ }^{2}$ Renal Division, Department of Medicine, Peking University First Hospital, Beijing, People's Republic of China; ${ }^{3}$ CardioCerebrovascular Control and Research Center, Institute of Basic Medicine, Shandong Academy of Medical Sciences, Jinan, Shandong, People's Republic of China; ${ }^{4}$ Renal Division, Department of Medicine, Peking University International Hospital, Beijing, China; ${ }^{5}$ National Institute of Health Data Science at Peking University, Beijing, People's Republic of China; ${ }^{6}$ Peking University, Center for Data Science in Health and Medicine, Beijing, People's Republic of China

*These authors contributed equally to this work
Correspondence: Luxia Zhang

Email zhanglx@bjmu.edu.cn
Purpose: In prior analyses, blood pressure (BP) was related to rapid kidney function decline (RKFD). However, studies of this relationship in populations of advanced age are lacking. In the present study, we therefore examined the relationship between BP and RKFD in a population of 284 hypertensive Chinese individuals over the age of 80 .

Patients and Methods: All study participants were diagnosed with hypertension (systolic BP [SBP] 160-200 mmHg; diastolic BP [DBP] <110 mmHg). RKFD was defined based upon a decline in estimated glomerular filtration rate (eGFR) $>5 \mathrm{~mL} / \mathrm{min}$ per $1.73 \mathrm{~m}^{2}$ per year during follow-up. The Cox regression models (competing risk models) were used for calculating hazard ratios (HRs) to examine the relationship between SBP, DBP, pulse pressure (PP) and RKFD.

Results: Over a 3.3-year median follow-up period, 68 study participants (23.9\%) were diagnosed with RKFD, while 35 (12.3\%) died. After adjusting for confounding variables, we determined that each $10 \mathrm{mmHg}$ rise in SBP and PP was associated with a $34 \%$ and $110 \%$ increase, respectively, in RKFD risk (adjusted HR: $1.34,95 \%$ confidence interval [CI]: 1.05-1.71 for SBP, $p=0.02$; HR: 2.10, 95\% CI: $0.87-5.08$ for PP, $p=0.10$ ). In addition, we determined that each $10 \mathrm{mmHg}$ increase in DBP was linked to a $10 \%$ reduction in RKFD risk (adjusted HR: $0.90,95 \%$ CI: $0.70-1.14, p=0.37$ ).

Conclusion: Our results indicate that SBP, but not DBP or PP, is positively correlated with RKFD risk in a very elderly hypertensive Chinese population.

Keywords: blood pressure, rapid kidney function decline, very elderly, China

\section{Introduction}

There is increasingly robust evidence that hypertension is a chronic kidney disease (CKD) risk factor and is strongly predictive of rapid kidney function decline (RKFD) in elderly individuals. ${ }^{1-3}$ To date, however, there have been very few longitudinal studies examining how hypertension relates to RKFD risk in very elderly adults (>80 years old) who are likely to suffer from both hypertension and CKD. ${ }^{4}$

In addition, while blood pressure (BP) is known to be associated with RKFD risk, how systolic BP (SBP), diastolic BP (DBP), and pulse pressure (PP) are specifically associated with this risk remains to be determined in very elderly populations. This is a clinically important consideration, as very elderly hypertensive patients often exhibit significantly increased SBP together with normal or low DBP. 
In light of these uncertainties, this study was designed to explore how individual BP components relate to RKFD risk among 284 very elderly hypertensive Chinese individuals.

\section{Patients and Methods}

\section{Participants}

Beginning in 2004, a total of 284 hypertensive patients > 80 years old (as confirmed by official documentation, and with no upper limit on age) from Shandong Province, China, were recruited for participation in a double-blind placebo-controlled trial exploring the risks and benefits of antihypertensive treatment. ${ }^{5-7}$ In this study, hypertension was defined as an SPB of 160-200 mmHg and a DBP of $<$ $110 \mathrm{mmHg}$ while resting in a seated position. Participants were excluded from this study if they suffered from accelerated hypertension, secondary hypertension, exhibited serum creatinine levels $\geq 150 \mathrm{~mol} / \mathrm{L}$, were diagnosed with dementia at study baseline. All 284 participants provided written informed consent, as detailed in previous reports. ${ }^{8,9}$ The Beijing Hypertension League Institute controlled participant randomization into the placebo and antihypertensive treatment groups. Participants were followed for 3 years following enrollment. Following trial completion in 2007, all participants were administered antihypertensive treatments and were followed for an additional year. The Ethics Committee of the Beijing Hypertension League Institute provided ethical approval for this study. ${ }^{5,9,10}$ All procedures performed in this study conform to the standards of the 1964 Helsinki Declaration and subsequent relevant ethics.

\section{Blood Pressure Measurement}

Baseline BP values in each participant were measured by trained staff who conducted three sitting BP readings using a random zero sphygmomanometer. The recorded value was the average of the last two readings. PP was defined as the difference between SBP and DBP.

\section{Covariate Analysis}

Patient demographic characteristics such as age, sex, smoking, alcohol consumption, and cardiovascular disease (CVD) history were ascertained by research staff using a questionnaire. The body weight and height of each participant were additionally measured and used to calculate body mass index (BMI) values as follows: $\mathrm{BMI}=$ weight $(\mathrm{kg}) /[\text { height }(\mathrm{m})]^{2}$. An automated biochemical analyzer was used to measure key blood biochemical parameters such as fasting glucose, uric acid levels, and total cholesterol levels. The mini-mental status exam (MMSE) was used to evaluate participant cognitive function.

\section{Assessment of Kidney Function}

A Hitachi 7600 automated biochemical analyzer (Hitachi, Tokyo, Japan) was used to measure participant serum creatinine levels at baseline and final follow-up using Jaffe's kinetic method. Estimated glomerular filtration rate (eGFR) values were calculated using a modified Modification of Diet in Renal Disease (MDRD) equation as described previously. ${ }^{11}$ An eGFR $>30 \mathrm{~mL} / \mathrm{min} / 1.73 \mathrm{~m}^{2}$ was noted for all the participants at baseline. RKFD was defined based upon a decline in estimated glomerular filtration rate $(\mathrm{eGFR})>5 \mathrm{~mL} / \mathrm{min}$ per $1.73 \mathrm{~m}^{2}$ per year during follow-up. The annual eGFR decline rate $=$ (the baseline eGFR - the last eGFR)/follow-up time. ${ }^{12}$

\section{Statistical Analysis}

Normally distributed continuous variables are given as means with standard deviations, whereas non-normally distributed continuous variables are given as medians with interquartile ranges (IQRs). Categorical variables are given as proportions. These variables were compared via analyses of variance (ANOVA), Kruskal-Wallis tests, $\chi^{2}$ tests, or Fisher's exact tests, as appropriate. The missing data is deleted.

The proportional hazard $(\mathrm{PH})$ assumptions were tested by the Schoenfeld residual scatter plot graph and Person correlation coefficients test. ${ }^{13}$ The relationship between specific BP parameters (SBP, DBP, and PP) and RKFD was assessed using the Cox $\mathrm{PH}$ or non-PH regression models (competing risk models). Data were adjusted based upon participant sex (male or female), age (in 1 year increments), smoking status (yes/no), CVD history (yes/no), BMI (in $1 \mathrm{~kg} / \mathrm{m}^{2}$ increments), total cholesterol (in $1 \mathrm{mmol} / \mathrm{L}$ increments), fasting blood glucose (in $1 \mathrm{mmol} / \mathrm{L}$ increments), eGFR (per $10 \mathrm{~mL} / \mathrm{min} / 1.73 \mathrm{~m}^{2}$ ), uric acid levels (in $\mathrm{mg} / \mathrm{dL}$ increments), MMSE scores (in 1 point increments), and antihypertensive treatment status (yes/no).

A two-tailed $\mathrm{P}<0.05$ was the significance threshold. SAS v 9.4 (SAS Institute Inc., USA) was used for statistical testing.

\section{Results}

This study population had a median age of 83.0 years (range: 81.4-85.2), and the participant population was 
Table I Baseline Characteristics of Participants

\begin{tabular}{|c|c|c|c|c|}
\hline Variables & Without RKFD & With RKFD & All-Cause Death & p-value \\
\hline Number of participants, $\mathrm{n}$ & 178 & 68 & 35 & \\
\hline Male, \% (n) & $22.5(40)$ & $30.9(21)$ & $31.4(I I)$ & 0.29 \\
\hline Age, years & $83.0(81.3-85.2)$ & $83.5(81.6-85.8)$ & $82.5(81.0-85.1)$ & 0.57 \\
\hline Current smoking, \% (n) & $6.2(11)$ & $5.9(4)$ & $20.0(7)$ & 0.03 \\
\hline History of CVD, \% (n) & $5.1(9)$ & $5.9(4)$ & $2.9(1)$ & 0.85 \\
\hline Antihypertensive treatment, \% (n) & $52.3(93)$ & $54.1(37)$ & $40.0(14)$ & 0.35 \\
\hline Body mass index, $\mathrm{kg} / \mathrm{m}^{2}$ & $22.7(21.3-23.6)$ & $22.3(21.0-23.3)$ & $22.7(21.5-24.1)$ & 0.12 \\
\hline Hemoglobin, g/dL & $12.0(\mid 1.1-12.9)$ & $12.0(11.2-13.7)$ & $11.7(10.9-13.3)$ & 0.49 \\
\hline Fasting blood glucose, $\mathrm{mmol} / \mathrm{L}$ & $5.0(4.4-5.5)$ & $4.8(4.2-5.3)$ & $5.0(4.6-5.3)$ & 0.15 \\
\hline Total cholesterol, $\mathrm{mmol} / \mathrm{L}$ & $4.7(4.3-5.1)$ & $4.7(4.1-5.0)$ & $4.7(4.2-5.3)$ & 0.12 \\
\hline Serum uric acid, mg/dL & $4.2(3.3-5.1)$ & $4.4(2.9-5.6)$ & $3.8(2.9-4.7)$ & 0.32 \\
\hline Systolic BP, mmHg & $173.0(165.0-185.5)$ & $178.0(166.0-190.0)$ & $175.0(165.0-185.0)$ & 0.38 \\
\hline Diastolic BP, mmHg & $87.0(78.0-92.0)$ & $81.0(78.0-88.0)$ & $86.0(78.0-93.0)$ & 0.10 \\
\hline Pulse pressure, $\mathrm{mmHg}$ & $89.0(81.0-98.5)$ & $94.0(87.0-104.0)$ & $91.0(81.0-99.0)$ & 0.04 \\
\hline Serum creatinine, $\mu \mathrm{mol} / \mathrm{L}$ & $108.0(97.0-126.0)$ & $70.0(64.5-79.5)$ & $107.0(92.0-123.0)$ & $<0.001$ \\
\hline eGFR, $\mathrm{mL} / \mathrm{min} / 1.73 \mathrm{~m}^{2}$ & $50.1(43.7-59.1)$ & $85.5(76.1-105.5)$ & $52.0(44.1-69.4)$ & $<0.001$ \\
\hline The MMSE score, point & $21.5(20-23)$ & $21.5(20.0-23.0)$ & $21.0(18.0-23.0)$ & 0.20 \\
\hline
\end{tabular}

Notes: The data are presented as percentage $(n)$ for categorical variables, mean \pm SD for continuous variables (normal distribution), median (IQR) for continuous variables (non-normal distribution).

Abbreviations: SD, standard deviation; RKFD, rapid kidney function decline; CVD, cardiovascular disease; BP, blood pressure; eGFR, estimated glomerular filtration rate.

26.1\% male. Participant follow-up was conducted for a median of 3.3 years (range: $2.2-4.0$ ), and 3 participants $(1.1 \%)$ were lost to follow-up. Over the study period, 68 (23.9\%) participants met the criteria for RKFD, and there were $35(12.3 \%)$ all-cause deaths.
Participant baseline characteristics are shown in Table 1. Participants with RKFD ( $n=68)$ had higher baseline PP and eGFR values relative to other participants $(n=178)$.

Figures 1-3, and Table 2 show that SBP and DBP were not time-varying variables, while $\mathrm{PP}$ was a time-varying

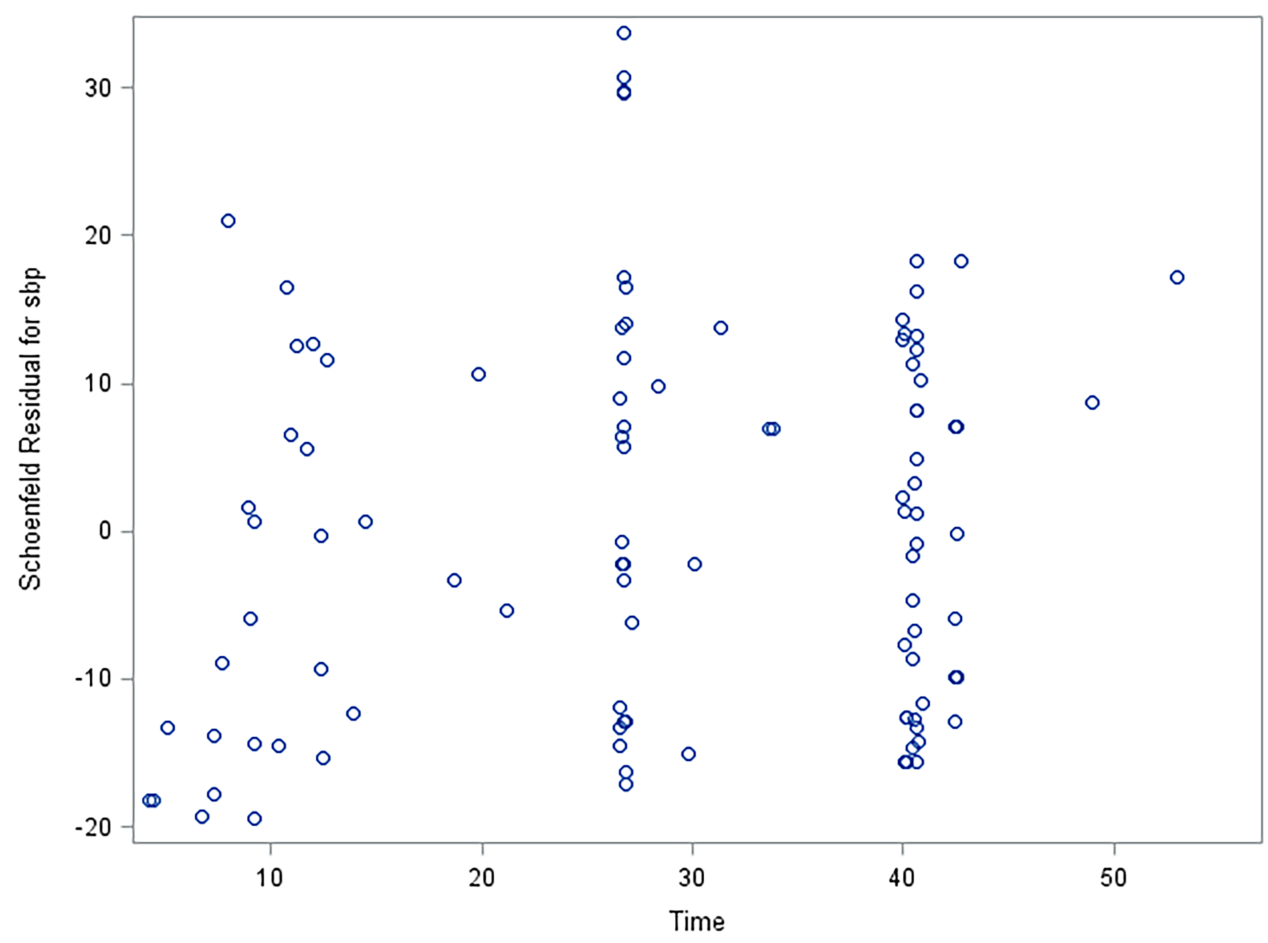

Figure I Schoenfeld residual for SBP with time. 


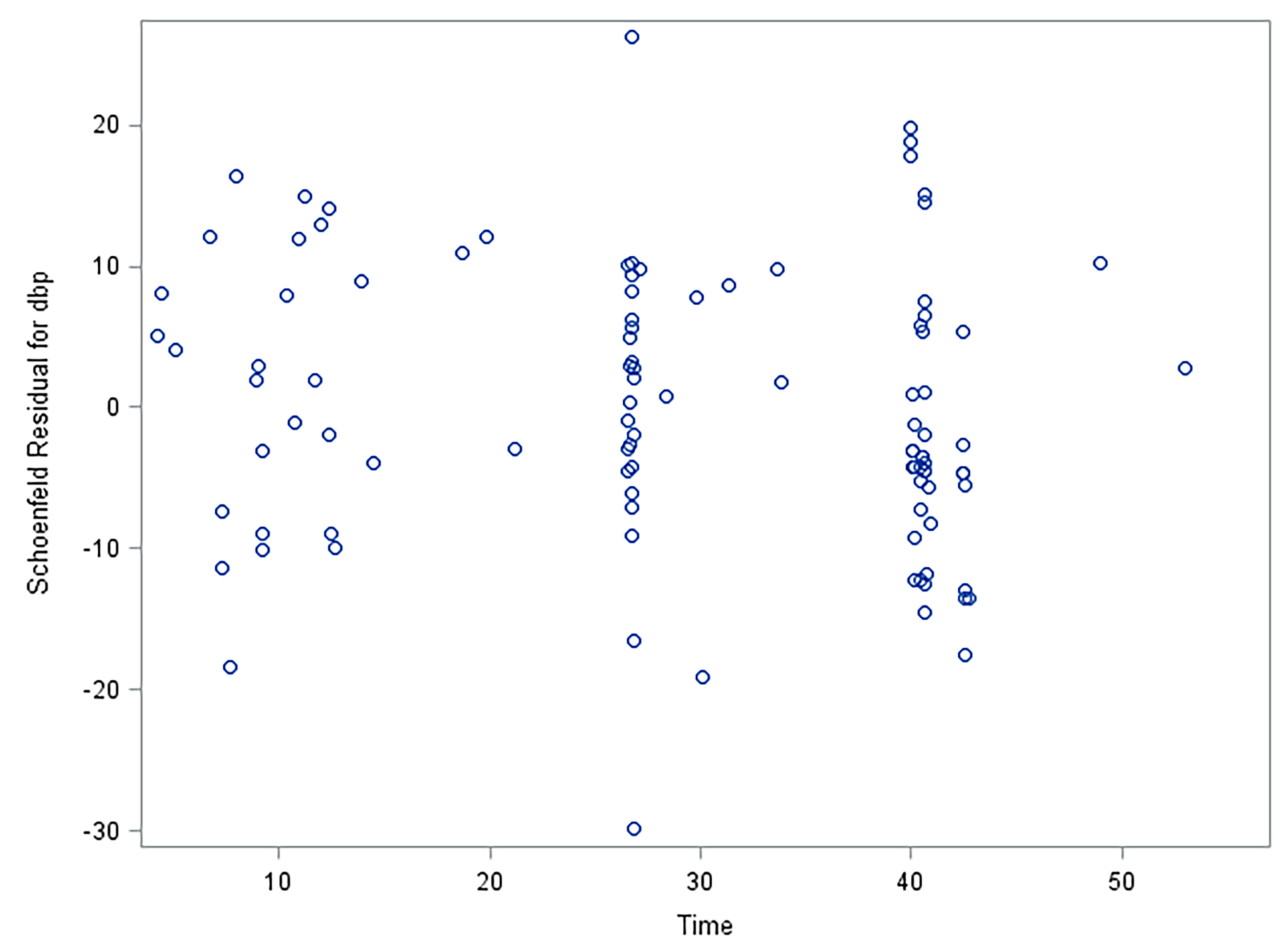

Figure 2 Schoenfeld residual for DBP with time.

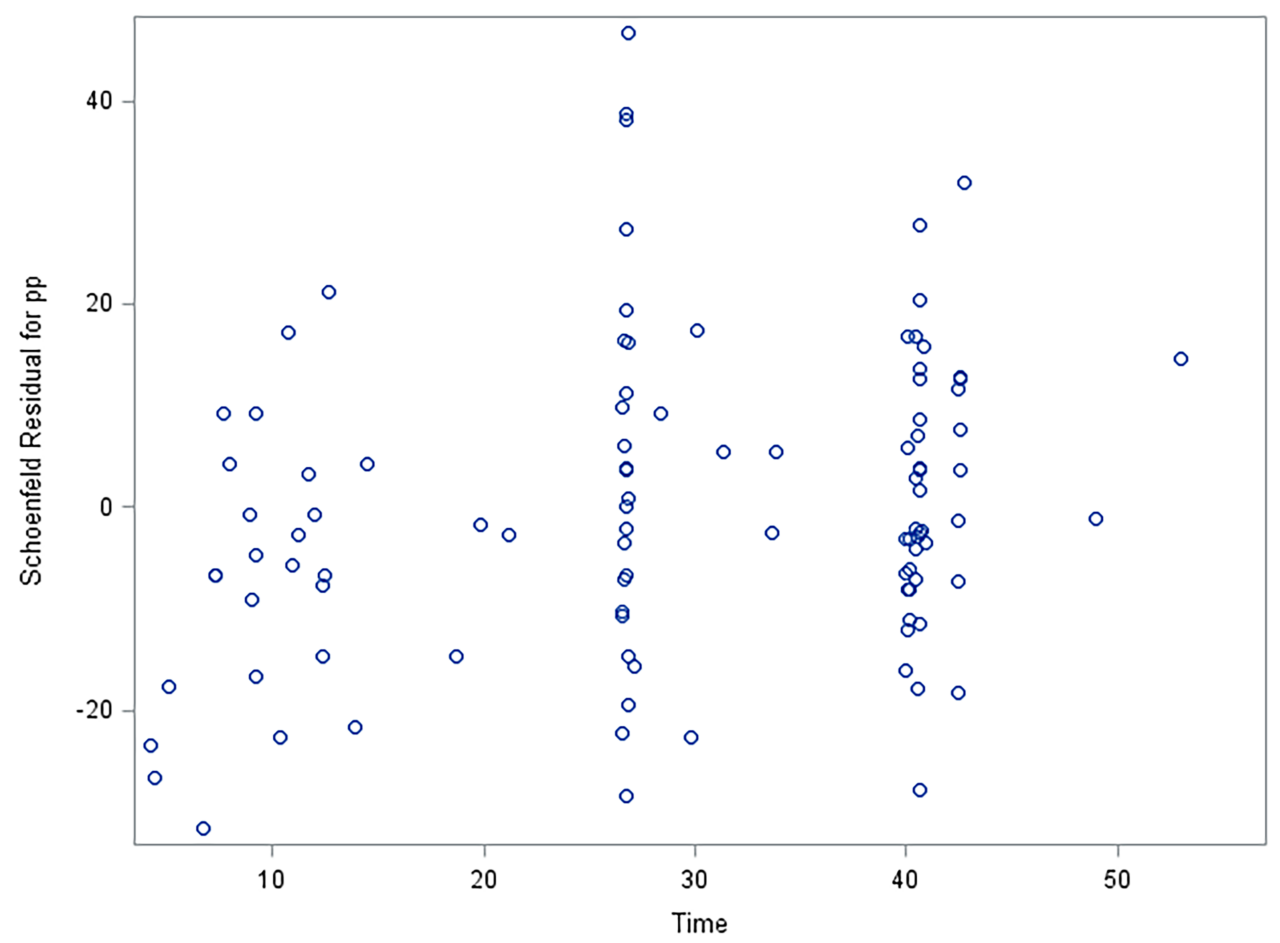

Figure 3 Schoenfeld residual for PP with time.

variable. We next conducted the Cox PH regression mod- models, by adding an interactive variable (PP*Time) ${ }^{14}$ els to examine the relationship between SBP, DBP and to examine the relationship between PP and RKFD risk RKFD risk; meanwhile, the Cox non-PH regression (Table 3). 
Table 2 Testing the Proportional Hazard Assumptions

\begin{tabular}{|l|l|l|l|}
\hline & $\begin{array}{l}\text { Systolic } \\
\text { BP }\end{array}$ & $\begin{array}{l}\text { Diastolic } \\
\text { BP }\end{array}$ & $\begin{array}{l}\text { Pulse } \\
\text { Pressure }\end{array}$ \\
\hline $\begin{array}{l}\text { Pearson correlation } \\
\text { p-value }\end{array}$ & 0.10 & -0.15 & 0.23 \\
\hline
\end{tabular}

Table 3 Baseline Blood Pressure and Rapid Kidney Function Decline

\begin{tabular}{|c|c|c|}
\hline & $\begin{array}{l}\text { Rapid Kidney } \\
\text { Function Decline }\end{array}$ & p-value \\
\hline \multicolumn{3}{|l|}{$\begin{array}{l}\text { Systolic BP, per } 10 \mathrm{mmHg} \\
\text { increase }\end{array}$} \\
\hline Crude HR & $1.27(1.02-1.57)$ & 0.03 \\
\hline Age, sex adjusted HR & $1.30(1.04-1.61)$ & 0.02 \\
\hline Multivariable adjusted $\mathrm{HR}^{\mathrm{a}}$ & $1.34(1.05-1.7 I)$ & 0.02 \\
\hline \multicolumn{3}{|l|}{$\begin{array}{l}\text { Diastolic BP, per } 10 \mathrm{mmHg} \\
\text { increase }\end{array}$} \\
\hline Crude HR & $0.70(0.05-0.89)$ & 0.004 \\
\hline Age, sex adjusted HR & $0.69(0.54-0.89)$ & 0.004 \\
\hline Multivariable adjusted $\mathrm{HR}^{\mathrm{a}}$ & $0.90(0.70-1.14)$ & 0.37 \\
\hline \multicolumn{3}{|l|}{$\begin{array}{l}\text { Pulse pressure, per } 10 \mathrm{mmHg} \\
\text { increase }\end{array}$} \\
\hline Crude HR & $1.77(0.78-3.91)$ & 0.16 \\
\hline Age, sex adjusted HR & $1.72(0.79-3.78)$ & 0.18 \\
\hline Multivariable adjusted $\mathrm{HR}^{\mathrm{a}}$ & $2.10(0.87-5.08)$ & 0.10 \\
\hline
\end{tabular}

Notes: Data are presented as HR $(95 \% \mathrm{Cl}) .{ }^{a}$ Model was further adjusted for age, sex, body mass index, current smoking, history of CVD, antihypertensive treatment, fasting glucose, total cholesterol, uric acid, the MMSE score and eGFR.

Abbreviations: BP, blood pressure; CVD, cardiovascular disease; MMSE, minimental state examination; eGFR, estimated glomerular filtration.

After adjusting for potential confounding variables, we found that each $10 \mathrm{mmHg}$ increase in SBP was correlated with a $34 \%$ increase in RKFD risk (adjusted hazards ratio [HR], 1.34; 95\% confidence interval [CI], 1.05-1.71; $p=0.02$ ). In addition, each $10 \mathrm{mmHg}$ increase in DBP was correlated with a $10 \%$ reduction in RKFD risk (HR, $0.90 ; 95 \% \mathrm{CI}, 0.70-1.14 ; p=0.37$ ), and each $10 \mathrm{mmHg}$ increase in PP was correlated with a $110 \%$ increase in RKFD risk (HR, 2.10; 95\% CI, 0.87-5.08; $p=0.10$ ).

\section{Discussion}

Our results indicate that SBP, but not DBP or PP, is an independent predictor of RKFD risk in a hypertensive Chinese population over the age of 80 . These results may aid clinicians in the identification of individuals at a high risk of developing RKFD.

There has been increasing recognition in recent years of the relationship between BP and RKFD. The
Cardiovascular Health Study ${ }^{1}$ found that elevated SPB and PP values were associated with increased RKFD risk in the elderly, whereas DBP values were unrelated to this risk, consistent with our results. Kim et al ${ }^{2}$ similarly found that PP was an independent predictor of RKFD incidence in a South Korean cohort of individuals that were 63.0 \pm 10.0 years old. Hirayama et $\mathrm{al}^{3}$ similarly found that SBP, but not DBP, was independently associated with rapid declines in eGFR among a Japanese cohort with an average age of $62.9 \pm 7.6$ years. These results clearly emphasize that only SBP is an important correlated for renal risk in elderly populations. These prior studies, however, had a significantly lower average age than did the present study. Furthermore, these studies did not include baseline kidney function in their multivariate models, even though baseline kidney function has been shown to be an RKFD risk factor, thus potentially confounding their results. Wang et $\mathrm{al}^{15}$ detected a dose-dependent relationship between baseline BP and RKFD in a middle-aged Chinese population even after controlling for basal eGFR and other confounding variables. Our results further extend these findings by demonstrating that a similar correlation exists in a very elderly Chinese population with an average age of $>80$ years. In contrast, Vaes et $\mathrm{al}^{16}$ found that elevated baseline SBP and PP values were associated with RKFD risk in individuals from 60 to 69 years old, whereas all measured BP parameters were unrelated to RKFD risk in individuals $>80$ years old. As such, further research is required in order to fully elucidate the relationship between BP and RKFD in the very elderly.

Multiple mechanisms have the potential to contribute to the observed relationship between hypertension and elevated RKFD risk. Hypertension-associated rises in intraglomerular pressure are common, ${ }^{17}$ and have the potential to induce or exacerbate glomerular and interstitial fibrosis. ${ }^{1}$ Such fibrosis can, in turn, drive renal ischemia and nephron injury. ${ }^{18}$ Hypertension-associated volume overload can also result in a reshaping of the afferent arteriole, ${ }^{17}$ resulting in increased arterial stiffness. ${ }^{2,19}$ Such stiffening can reduce arterial elasticity and result in poor vasodilation and vasoconstriction. ${ }^{20-22}$ Reduced nephron blood flow can result in these pathophysiological changes. ${ }^{23}$ While these mechanisms are promising, additional research will be required to fully understand the mechanistic basis of our findings.

This study has multiple strengths and limitations that should be taken into consideration. The primary strength of this study is the fact that it examined the relationship 
between BP parameters and RKFD risk in a rarely studied patient population by controlling for a number of potential confounding variables. However, this study is limited by its small sample size and the fact that the majority of study participants were female. According to reports form Lancet, ${ }^{24}$ women live with significant higher estimated average healthy life expectancy than men do. Just as the results and tables shown in this manuscript, it makes sense that the majority of participants were female. Therefore, there was a survivor bias of our study, which might lower the statistical power. In addition, all study participants were recruited from a single Chinese Province, and these results may therefore not be generalizable to other populations. However, these factors likely led to increased homogeneity with respect to the living conditions and medical care received by these study participants, thereby increasing the internal validity of this analysis. Third, information regarding the participants' blood pressure measurement during the follow-up was missing. Fourth, hypertension was defined as an SBP of 160-200 mmHg and a DBP of $<110 \mathrm{mmHg}$ in our study, these results may therefore not be generalizable to all hypertensive population aged over 80 years. Fifth, information regarding the participants' other comorbidities status, socioeconomic status, medication use and the urine albumin value was not available. Sixth, the Jaffe's kinetic method was used for measurement of serum creatinine level, rather than the standardized isotope dilution mass spectrometry. ${ }^{25}$ Lastly, no favorable methods for estimating kidney function in the very elderly are available, thus constraining this analysis.

\section{Conclusion}

Our results suggest that SBP is an independent predictor of RKFD risk among a very elderly hypertensive Chinese population, whereas DBP is not predictive of such risk in these individuals. These findings emphasize the importance of appropriately evaluating and managing hypertension and kidney function among the very elderly.

\section{Ethical Statement}

All procedures performed in this study were in accordance with the ethical standards of the institutional and/or national research committee and with the 1964 Helsinki declaration and its later amendments or comparable ethical standards.

\section{Funding}

This work was supported by Grants from the National Natural Science Foundation of China (Grant Nos. 81771938,
91846101, 81301296), from Peking University (Grant Nos. BMU2018MX020, PKU2017LCX05), the National Key Technology R\&D Program of the Ministry of Science and Technology of the People's Republic of China (2016YFC1305400), and the University of Michigan Health System-Peking University Health Science Center Joint Institute for Translational and Clinical Research (BMU20160466, BMU2018JI012, BMU2019JI005).

\section{Disclosure}

The authors report no conflicts of interest in this work.

\section{References}

1. Rifkin DE, Katz R, Chonchol M, et al. Blood pressure components and decline in kidney function in community-living older adults: the Cardiovascular Health Study. Am J Hypertens. 2013;26(8):10 37-1044. doi:10.1093/ajh/hpt067

2. Kim CS, Kim HY, Kang YU, et al. Association of pulse wave velocity and pulse pressure with decline in kidney function. $J$ Clin Hypertens (Greenwich). 2014;16(5):372-377. doi:10.1111/jch.12302

3. Hirayama A, Konta T, Kamei K, et al. Blood pressure, proteinuria, and renal function decline: associations in a large community-based population. Am J Hypertens. 2015;28(9):1150-1156. doi:10.1093/ajh/ hpv003

4. Maw TT, Fried L. Chronic kidney disease in the elderly. Clin Geriatr Med. 2013;29(3):611-624. doi:10.1016/j.cger.2013.05.003

5. Bai K, Pan Y, Lu F, Zhao Y, Wang J, Zhang L. Kidney function and cognitive decline in an oldest-old Chinese population. Clin Interv Aging. 2017;12:1049-1054. doi:10.2147/CIA.S134205

6. Bai K, Pan Y, Lu F, Zhao Y, Wang F, Zhang L. Cognitive function and 3-year mortality in the very elderly Chinese population with chronic kidney disease. Clin Interv Aging. 2018;13:2055-2060. doi:10.2147/CIA.S173696

7. Chen R, Bai K, Lu F, et al. Electrocardiographic left ventricular hypertrophy and mortality in an oldest-old hypertensive Chinese population. Clin Interv Aging. 2019;14:1657-1662. doi:10.2147/ CIA.S218106

8. Bulpitt C, Fletcher A, Beckett N, et al. Hypertension in the Very Elderly Trial (HYVET): protocol for the main trial. Drugs Aging. 2001;18(3):151-164. doi:10.2165/00002512-200118030-00001

9. Beckett NS, Peters R, Fletcher AE, et al. Treatment of hypertension in patients 80 years of age or older. $N$ Engl J Med. 2008;358 (18):1887-1898. doi:10.1056/NEJMoa0801369

10. Liu LS, Wang JG, Shu-Ping M, et al. Characteristics of the Chinese subjects entered the hypertension in the very elderly trial. Chin Med $J$ (Engl). 2008;121(16):1509-1512. doi:10.1097/00029330-200808 020-00002

11. Ma YC, Zuo L, Chen JH, et al. Modified glomerular filtration rate estimating equation for Chinese patients with chronic kidney disease. J Am Soc Nephrol. 2006;17(10):2937-2944. doi:10.1681/ASN.20060 40368

12. Stevens PE, Levin A. Evaluation and management of chronic kidney disease: synopsis of the kidney disease: improving global outcomes 2012 clinical practice guideline. Ann Intern Med. 2013;158 (11):825-830. doi:10.7326/0003-4819-158-11-201306040-00007

13. Stensrud MJ, Hernan MA. Why test for proportional hazards? JAMA. 2020;323(14):1401. doi:10.1001/jama.2020.1267

14. Zhang Z, Reinikainen J, Adeleke KA, Pieterse ME, Groothuis-Oudshoorn CGM. Time-varying covariates and coefficients in Cox regression models. Ann Transl Med. 2018;6(7):121. doi:10.21037/atm.2018.02.12 
15. Wang Q, Xie D, Xu X, et al. Blood pressure and renal function decline: a 7-year prospective cohort study in middle-aged rural Chinese men and women. J Hypertens. 2015;33(1):136-143. doi:10.1097/HJH.0000000000000360

16. Vaes B, Beke E, Truyers C, et al. The correlation between blood pressure and kidney function decline in older people: a registry-based cohort study. BMJ Open. 2015;5(6):e007571. doi:10.1136/bmjopen2015-007571

17. Yoshioka T, Rennke HG, Salant DJ, Deen WM, Ichikawa I. Role of abnormally high transmural pressure in the permselectivity defect of glomerular capillary wall: a study in early passive Heymann nephritis. Circ Res. 1987;61(4):531-538. doi:10.1161/01.RES.61.4.531

18. Schnaper HW. The tubulointerstitial pathophysiology of progressive kidney disease. Adv Chronic Kidney Dis. 2017;24(2):107-116. doi:10.1053/j.ackd.2016.11.011

19. Wang MC, Tsai WC, Chen JY, Huang JJ. Stepwise increase in arterial stiffness corresponding with the stages of chronic kidney disease. Am J Kidney Dis. 2005;45(3):494-501. doi:10.1053/j. ajkd.2004.11.011

20. London GM, Guerin AP, Marchais SJ, Metivier F, Pannier B, Adda H. Arterial media calcification in end-stage renal disease: impact on all-cause and cardiovascular mortality. Nephrol Dial Transplant. 2003;18(9):1731-1740. doi:10.1093/ndt/gfg414

21. Goldsmith D, Ritz E, Covic A. Vascular calcification: a stiff challenge for the nephrologist: does preventing bone disease cause arterial disease? Kidney Int. 2004;66(4):1315-1333. doi:10.1111/ j.1523-1755.2004.00895.x
22. Hujairi NM, Afzali B, Goldsmith DJ. Cardiac calcification in renal patients: what we do and don't know. Am J Kidney Dis. 2004;43 (2):234-243. doi:10.1053/j.ajkd.2003.10.014

23. Vashishtha D, McClelland RL, Ix JH, Rifkin DE, Jenny N, Allison M. Relation between calcified atherosclerosis in the renal arteries and kidney function (from the multi-ethnic study of atherosclerosis). Am J Cardiol. 2017;120(8):1434-1439. doi:10.10 16/j.amjcard.2017.07.020

24. GBD 2016 DALYs and HALE Collaborators. Global, regional, and national disability-adjusted life-years (DALYs) for 333 diseases and injuries and healthy life expectancy (HALE) for 195 countries and territories, 1990-2016: a systematic analysis for the Global Burden of Disease Study 2016. [published correction appears in Lancet. 2017 Oct 28;390(10106):e38]. Lancet. 2017;390(10100):1260-1344. doi:10.1016/S0140-6736(17)32130-X

25. Levey AS, Coresh J, Greene T, et al. Expressing the modification of diet in renal disease study equation for estimating glomerular filtration rate with standardized serum creatinine values. Clin Chem. 2007;53(4):766-772. doi:10.1373/clinchem.2006.077180
Clinical Interventions in Aging

\section{Publish your work in this journal}

Clinical Interventions in Aging is an international, peer-reviewed journal focusing on evidence-based reports on the value or lack thereof of treatments intended to prevent or delay the onset of maladaptive correlates of aging in human beings. This journal is indexed on PubMed Central, MedLine, CAS, Scopus and the Elsevier

\section{Dovepress}

Bibliographic databases. The manuscript management system is completely online and includes a very quick and fair peer-review system, which is all easy to use. Visit http://www.dovepress.com testimonials.php to read real quotes from published authors. 